\title{
A Passively Morphing Trailing Edge Concept for Sailing Hydrofoil
}

\author{
Francis Hueber ${ }^{1}$, Giulia Caponnetto ${ }^{2}$ and Carlo Poloni ${ }^{3, *}$ \\ 1 Caponnetto-Hueber S.L., Calle Poeta Querol 1, Puerta 1, 46002 Valencia, Spain; \\ francis@caponnetto-hueber.com \\ 2 HUINDI, c/o Caponnetto-Hueber S.L., Calle Poeta Querol 1, Puerta 1, 46002 Valencia, Spain; \\ giulia.caponnetto@huindi.com \\ 3 Dipartimento di Ingegneria e Architettura, University of Trieste, Piazzale Europa 134142 Trieste, Italy \\ * Correspondence: poloni@units.it; Tel.: +395583808
}

\begin{abstract}
Sailing sports are experiencing a period of radical innovations. Traditional displacing monohulls have always been the reference for yachting in the past, but the continuous quest for performances has generated many variations and many convergences with surprising results. On the aerodynamic side conventional soft sails have been sometime replaced by rigid wings, but the biggest innovation has been the use of traction kites as a propulsion device. On the hydrodynamic side we have seen the rapid growth of hydrofoils, an old concept that is having a rebirth. Probably the most remarkable expression of innovation and integration between concepts having different roots is the hydrofoil kite-board, point of encounter of the traditional wave surfing, the traction kite innovation and the hydrofoil technology. Hydrofoil kite-board can reach speeds up to 3 times the wind speed, are at least one order of magnitude cheaper than any boat with comparable performances, easy to manage and races are spectacular. Hydrofoil are object of investigation trying to further improve performances as well as to increase the stability and the "sailability". In the following we will present a concept that extends the range of efficiency of the foil through a completely passive morphing of the wing.
\end{abstract}

Keywords: sailing; hydrofoil; kite-board

\section{Introduction}

Sailing hydrofoil are not an original concept. The first application is probably the Gordon Baker Monitor (1955) [1] while the James Grogono experimental catamarans Icarus (1972) followed by about a decade [2]. Since then, sailing hydrofoils remained confined to the niche of the inventors and mainly for the quest of the sailing speed record, until the development of stable foil concept for the International Moth Class (2004 circa) made foiling a commercially viable product [3]. This also gave the impulse, starting about in 2010, to experiment high tech hydrofoil on the large high performance catamarans of the $34^{\text {th }}$ edition of the America's Cup.

In the same years but on a completely different path, traditional wave surfing evolved in the branch of the windsurfing, a sport that became extremely popular in the ' 80 and ' 90 . Of the mid '70 are the first attempts to replace a sail with a kite on a catamaran (Jacob's Ladder, 1978) [4] a concept that became mature in the late ' 90 , when replacing a sail with a kite on a windsurf gave birth to the kiteboarding sport. Finally, adding a hydrofoil to a kite-board, we obtained the hydrofoil-kite-board, at present the most performant and innovative commercial sailing craft. Hydrofoil kiteboarding is having a rapid growth between professional and amateur riders and no other boat or board can give comparable sensations of speed. At the same time, it is in absolute affordable compared to any existing boat, while the small dimension of the board, hydrofoil and kite when folded make it easily transportable. 


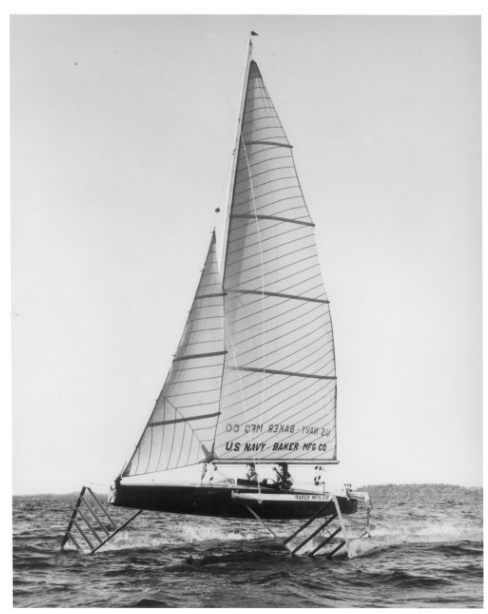

(a)

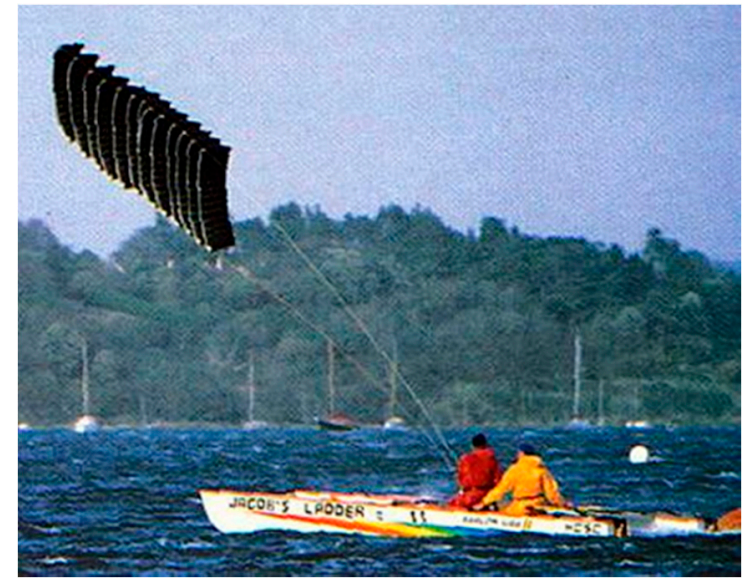

(c)

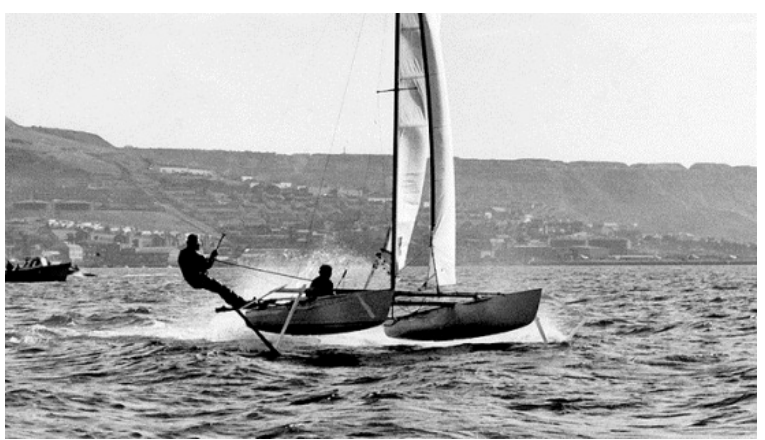

(b)

Figure 1. Pioneering hydrofoil sailing/kite boats: (a) Gordon Baker, Monitor (1955); (b) James Grogono, Icarus (1972); (c) Jacob's ladder (1978).

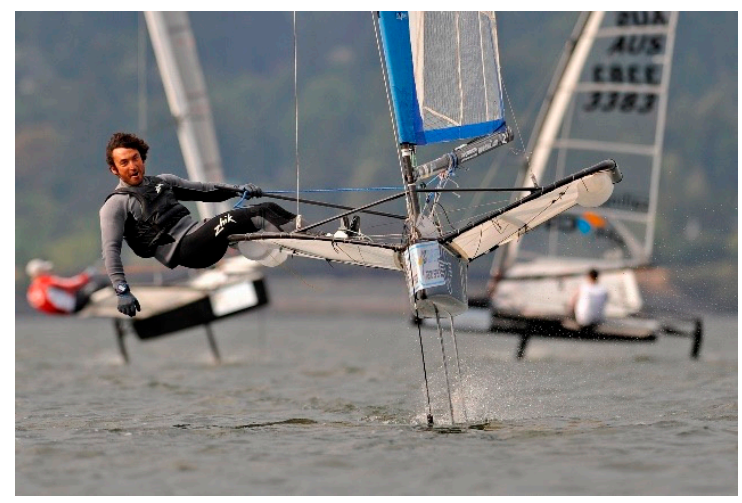

(a)

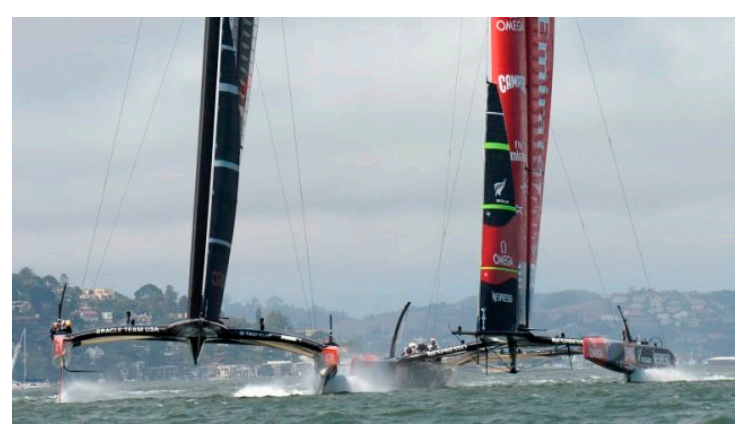

(b)

Figure 2. Hydrofoil sailing boats: (a) International Moth class (2004); (b) America's Cup (2013);

Hydrofoil producers are working to improve and to optimize the concept; from one side they are developing more stable foils that are easier to sail especially for the beginners. At the same time there is the quest for the highest possible performances. 
In this paper a new concept for improving stability and performance of a race hydrofoil-kite-board will be presented. The concept exploit the possibility of having a passively morphing trailing edge of the foil to adapt the section shape to speed.

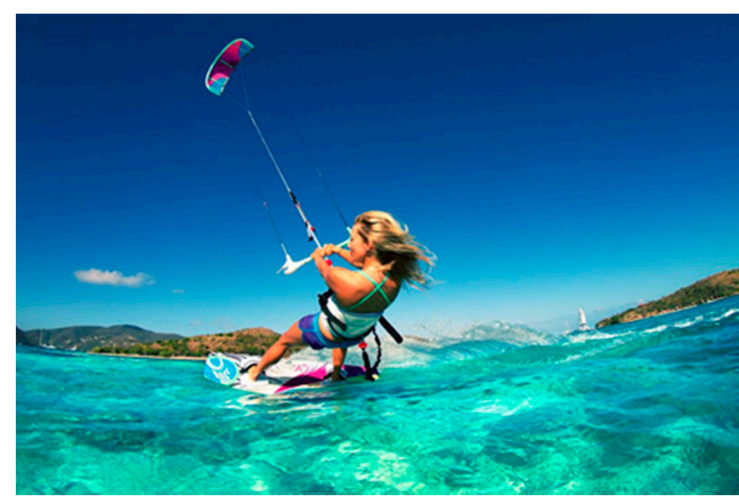

(a)

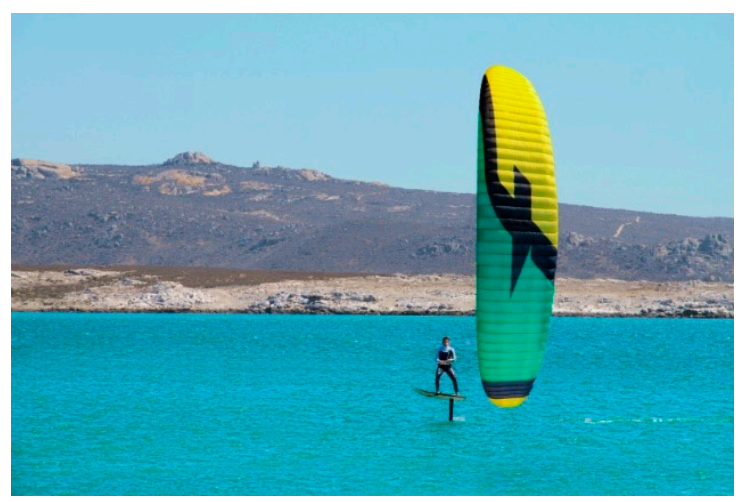

(b)

Figure 3. Kite boards: (a) simple kite-board; (b) hydrofoil-kite-board;

\section{Hydrofoil design}

A hydrofoil for kiteboard is made of four principal parts as described in fig.4a. A vertical strut that connects the board to the fuselage, the main wing at the forward extremity of the fuselage and a tail at the back. In normal sailing conditions, very little lift is generated by the strut. The duty of developing the lift that counteract the weight of the rider and the side force generated by the kite is taken by the main wing. The small tail at the back has a pitch stabilizing effect and most of the time generates a downward lift unfavorable for speed performances.

The main wing must be designed to operate in a wide range of conditions while fulfilling different constraints. To explain some of the design problems, we will do several simplifying hypotheses without losing generality.

Figs. $4 \mathrm{~b}$ and $4 \mathrm{c}$ show typical airfoil characteristics in term of lift and drag coefficients as a function of angle of attach and value of the camber ratio.

If we assume that the lift developed by the main wing $\mathrm{L}$ remains constant across the whole range of speed $\mathrm{V}$, the lift coefficient $\mathrm{Cl}$ will vary as shown in fig. $4 \mathrm{~d}$ (working condition). The foil will operate between the so-called takeoff speed (Vto) and the design maximum speed (Vmax). The bigger the range Vto-Vmax is, the larger will be the range of $\mathrm{Cl}$ the foil will experience.

There is a maximum $\mathrm{Cl}$ value at which a foil can operate at takeoff. Above this value the foil will stall. Even before stall a foil in water will cavitate. In both cases the foil won't be able to produce any more lift in an efficient way.

In general, if we chose the foil area for a very low takeoff speed, when sailing at higher speed we will have an excess of wetted surface dragging our foil. If we reduce the foil area to improve high speed performances, the takeoff speed will be too high. Therefore, different foil areas should be chosen for different range of wind speed.

In analogy with the wing of an airplane, we would like to adjust the surface and the shape of the wing between takeoff and cruise speed, something that is practically impossible in the case of small size hydrofoil construction. What is proposed in this paper is the possibility to modify the camber of the foil, namely the curvature of the section, in accordance with the speed requirement and without the direct intervention of the rider but in a passive way. 


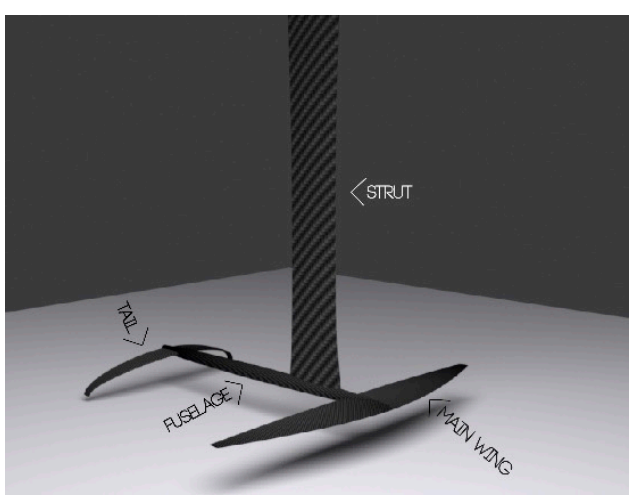

(a)

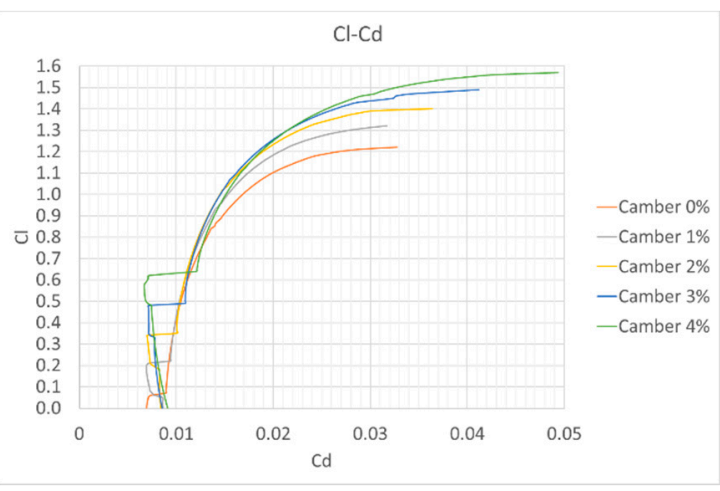

(c)

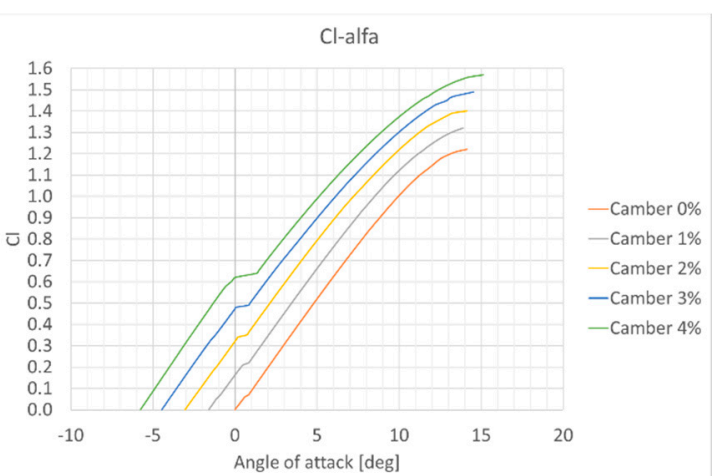

(b)

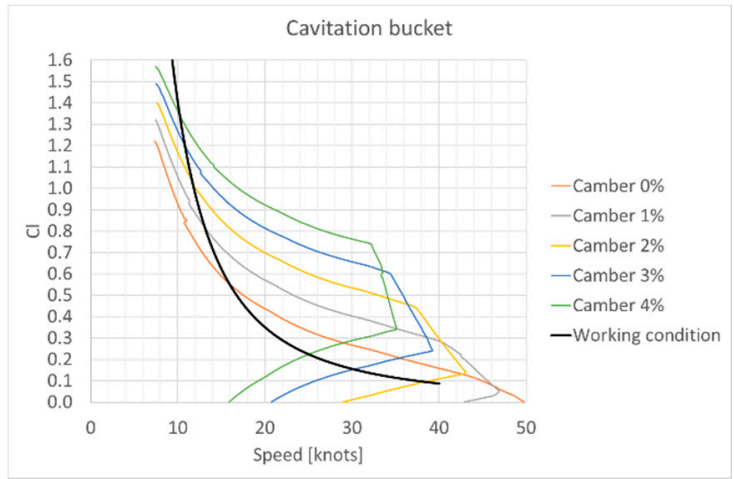

(d)

Figure 4. hydrofoil characteristics: (a) schematic assembly of a kite-board hydrofoil; (b) lift coefficient of the wing as a function of the angle of attach and for different camber values; (c) polar curves (lift coefficient versus drag coefficient for different camber values; (d) working condition line superimposed to the lift coefficient of the wing as a function of the speed considering also the lift limitation due to cavitation.

\subsection{Basic concept and passive flap analogy}

We can envisage the possibility of having a completely passive device to adapt the amount of camber with speed looking at the analogy with a symmetric foil having a plane flap at its trailing edge connected with a torsional spring (fig. 5a).

If the flap length is relatively small compared to the total foil length (let's say 30/20\%) any variation of angle of attack has a small effect on the pressure distribution over the flap surface. Fig 6 (rearranged from [5]) shows the effect on the chord wise aerodynamic load due a pure variation of angle of attack. Most of the load increase occurs at the leading edge of the foil $(x / C=0)$ and it is rapidly reducing going toward the trailing edge $(x / C=1)$ where the load goes to zero to fulfill the so called Kutta condition.

We assume that the torsional spring at the hinge of the flap is at rest when the flap angle to foil chord line is Beta_o and the torsional (linear) stiffness of the spring is Ke. When the flap is at any other angle Beta the spring will react with an elastic moment given by:

$$
M_{e}=K_{e}\left(\beta_{o}-\beta\right)
$$


If we assume that the effect of the angle of the attack is negligible over the length of the flap we can write the hydrodynamic moment at the hinge of the flap as:

$$
M_{h}=K_{h} \beta V^{2}
$$

where $\mathrm{Kh}$ is a constant and $\mathrm{V}$ is the flow speed.

The hydro elastic equilibrium condition ( $\mathrm{Me}=\mathrm{Mh}$ ) supplies the following relationship for actual flap angle:

$$
\beta=\frac{\beta_{o}}{1+\left(\frac{K_{h}}{K_{e}}\right) V^{2}}
$$

At $\mathrm{V}=0$ the flap deflection is Beta_o and as the speed increases Beta goes toward zero as $1 / \mathrm{V}^{\wedge} 2$. The softer the spring (small Ke) the more the flap will deflect for the same speed.

Although we used many simplifying hypotheses not adequate for a foil design, formula (3) gives a qualitative explanation of the concept. In particular, it shows the possibility to reduce the curvature close to the trailing edge of the foil (here the flap angle) with speed increases just relying on the pressure load naturally developed over the foil surface, namely in a passive way.

\subsection{Flexible trailing edge concept}

While for some applications we could design a passive flap device as the one shown above, we can retain the basics of the concept replacing the concentrated torsional spring with a distributed elasticity along the whole flap. This is particularly desired when we are concerned with the drag and efficiency of the section, and we try to maintain the smoothness of the surface instead of creating a kink as it happens with the hinged flap.

Fig. $5 \mathrm{~b}$ shows the schematics of the flexible trailing edge concept. The front part of the section (about 60 to $80 \%$ of the total chord) is virtually rigid as for any conventional foil. In the picture, the trailing edge if composed by layers of structural and flexible material (carbon or fiberglass) located on the upper surface of the foil, while the lower surface is made of filler with very low stiffness and specific damping properties. In alternative, the whole flexible trailing edge could be built with an isotropic material with equivalent mechanical properties of the laminate.

The mould shape (undeformed foil) must be designed in conjunction with the laminate stiffness in order to allow the desired deformation of the trailing edge under the hydrodynamic loads experienced at different speeds. This task is normally fulfilled using a fluid structure interaction (FSI) design method.

An example of the design procedure is presented below. 


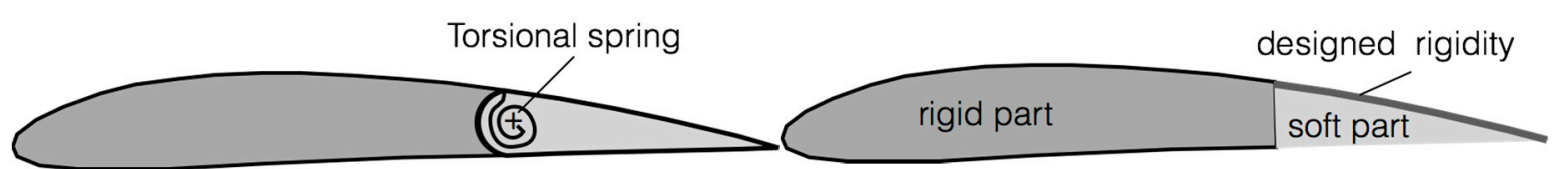

(a)

(b)

Figure 5. Passive flap: (a) Torsional spring concept; (b) Flexible trailing edge concept;

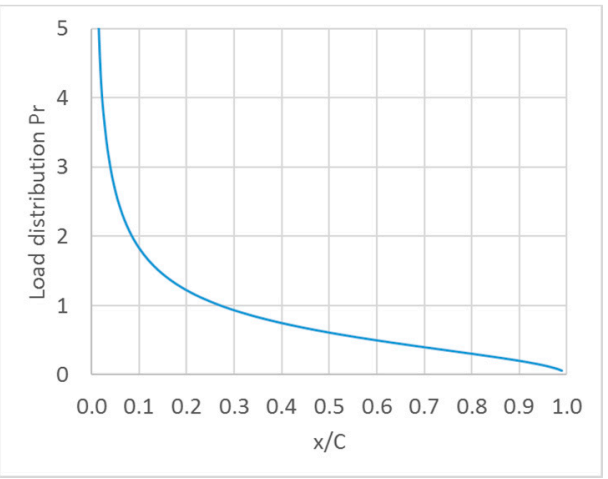

Figure 6. The chordwise load distribution due to pure angle of attack variation. At $x / C=1$ (trailing edge) the effect is vanishing, while it is maximum near to the $\mathrm{x} / \mathrm{C}=0$ (leading edge).

\section{Design of a flexible trailing edge}

In this paragraph, we describe the process to determine the stiffness characteristics of the flexible trailing edge once two distinct sailing points (P1 and P2) are given. Normally we'll chose P1 at takeoff speed V1 (lowest foiling speed) and P2 at another speed close to the top speed V2.

If the quantities V1, the total load acting on the foil and the lift coefficient $\mathrm{Cl} 1$ at $\mathrm{P} 1$ are given, we can determine the required foil area and from assumptions about the span we obtain the mean chord. In the example the chord length is $\mathrm{C}=0.1 \mathrm{~m}$.

We assume a takeoff speed V1=10 kn and a corresponding $\mathrm{Cl} 1 \mathrm{of} 1.4$. At this condition, we want to avoid stall and leading edge cavitation on the upper surface of the foil that would create an excess of drag and eventually loss of lift.

We fix our top speed to $\mathrm{V} 2=35 \mathrm{kn}$; if the load over the foil is the same at the two speeds (simplifying hypothesis) we will have $\mathrm{Cl} 2=\mathrm{Cl} 11^{*}(\mathrm{~V} 1 / \mathrm{V} 2)^{\wedge} 2=0.115$. At $\mathrm{V} 2$ we want to be free from any cavitation and having a low drag coefficient.

Figure 7 shows 2 sections (S1 and S2) having the same thickness distribution all along the chord, same camber distribution from the leading edge to $60 \%$ of the chord and two different camber distribution from $60 \%$ to the very end of the trailing edge. These two sections are compatible with a single foil having a flexible trailing edge along the rear $40 \%$ of the chord. If we compute the aerodynamic characteristics of the two foil, we can obtain the cavitation bucket of figure $7 \mathrm{~b}$. It is shown that at $\mathrm{V} 1$ and $\mathrm{Cl} 1$ we are just below the leading edge cavitation inception curve for section $\mathrm{S} 1$ (top orange line), fulfilling our first design requirement at P1. At V2 and $\mathrm{Cl} 2$ we are free from any cavitation with the section S2, as we are well inside the grey lines that separates the cavitating from the non cavitating regions. 


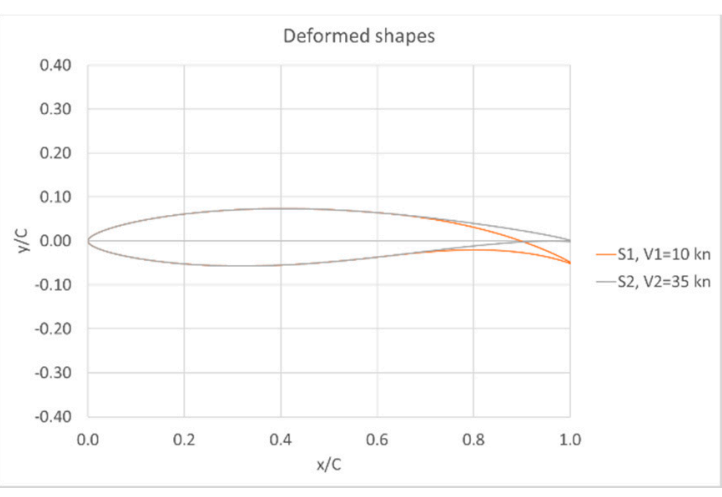

(a)

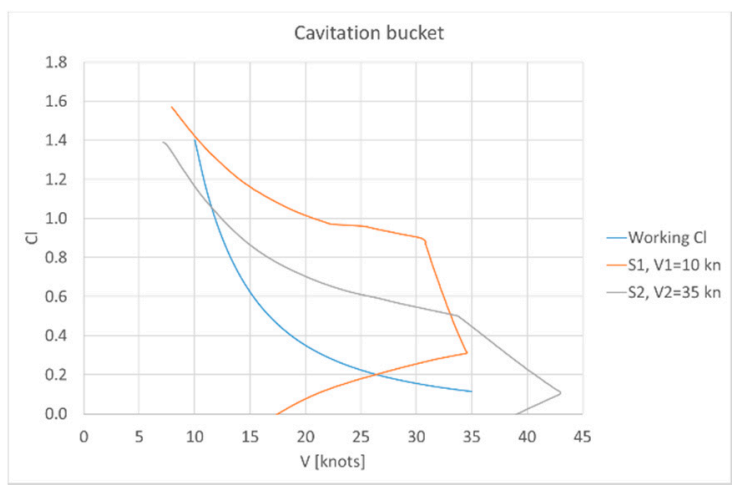

(b)

Figure 7. Deformable airfoil: (a) wing section at take-off speed and at maximum speed; (b) Lift coefficient of the two sections as a function of the operating speed;

We can also observe that at the V1/Cl1 working point we should have leading edge cavitation on the upper side with the less cambered sections S2 (the point is above the grey line) and at $\mathrm{V} 2 / \mathrm{Cl} 2$ leading edge cavitation on the lower side with the S1 section, as the point is below the orange line. In general, it will be difficult to find a single "undeformable" section shape able to fulfill the design requirements in the whole range of sailing conditions or, if feasible, the drag characteristic would be compromised. From this it follows the potential advantage of having a morphing flexible trailing edge.

If we are satisfied with the two foil shapes, the problem now is to determine the trailing edge mechanical characteristics that, starting from a still unknown undeformed or "mould" shape, will matches the S1 shape at $\mathrm{V} 1 / \mathrm{Cl} 1$ and the S2 shape at $\mathrm{V} 2 / \mathrm{Cl} 2$.

Fig. 8a shows how the pressure distribution over S1 and S2 looks like at the respective design points. It must be noticed that the area contained by the two curves is identical, as it represents the value of the sectional lift in the two conditions, that we have assumed to be the same. Nevertheless, the chord wise distribution is very different. At take off the dynamic pressure is small; a large angle of attack is needed (despite the large camber) and the load is concentrated toward the leading edge. At high speed the dynamic pressure is 12.5 time larger; a much smaller angle of attack (or a negative angle of attack) is required to produce the same lift and, even with a smaller camber, the front part of the section will produce negative lift, that will be compensated by a large load toward the trailing edge.

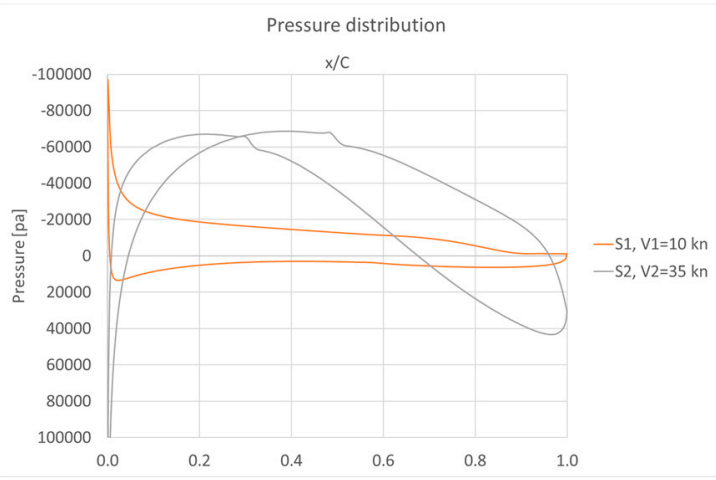

(a)

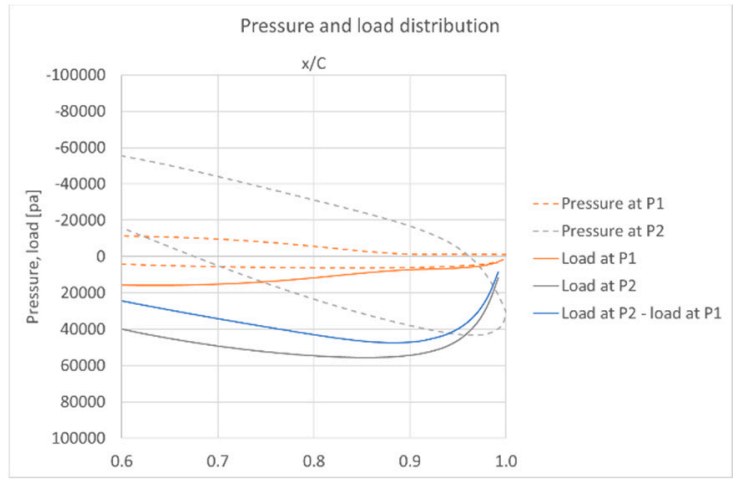

(b)

Figure 8. Deformable airfoil: (a) pressure distribution at take-off speed and at maximum speed; (b) pressure distribution and load distribution in the rear part of the profile; 
In the rear $40 \%$ of foil, in way of the flexible structure, the pressure load at $35 \mathrm{kn}$ is much higher than at $10 \mathrm{kn}$. This is consistent with the fact that increasing speed the trailing edge will bend upward, then reducing the camber. For our scope, we are more interested in the load distribution than in the pressure distribution, where for load we mean the pressure difference between the two opposite surfaces of the foil at any chordwise position. Is the load that will deform the trailing edge; and is the difference of load distributions between the two working conditions P1 and P2 that determines the variation of camber between S1 and S2.

The load distribution along the flexible trailing edge at P1 and P2 and the variation of load between $\mathrm{P} 1$ and $\mathrm{P} 2$ is shown in fig. $8 \mathrm{~b}$.

The camber of the flexible trailing edge at P1 and P2, as well as the variation of camber between P1 and P2 are illustrated in figure 9.

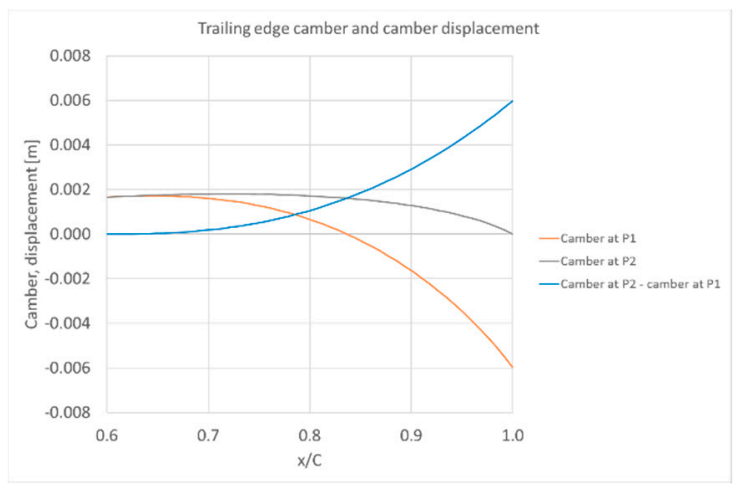

Figure 9. Camber variation at the two operation conditions

To simplify the exposition of the problem, but without losing generality, we can assume again some simplifying hypothesis. We consider the flexible trailing edge structure as a thin beam clamped at one edge and with linear elastic property. The modulus and inertia (and the EI value) can vary along the beam, the loads are always orthogonal to the beam and are small. To go further with the simplification, we can discretize the beam with $\mathrm{N}$ segments, each segment $\mathrm{i}(\mathrm{i}=1, \mathrm{~N})$ having a fixed value EI_i and a constant load distribution over the segment or a force F_i located at the center of the segment.

Now the problem is reduced to find the $\mathrm{N}$ values EI_i that give the vertical displacement $\mathrm{Y}_{-} \mathrm{i}$ (the discretize variation of camber P2-P1) under the load F_i. The solution of this problem is straightforward. For our example, we have considered a mean modulus of the structure of 7.5E10 Pa and a minimum thickness admissible of $0.2 \mathrm{~mm}$ ( 1 ply of fiber). The EI (per meter span obtained) is shown in fig. 10a and in fig. $10 \mathrm{~b}$ is shown the thickness variation of the flexible trailing edge structure. In the example we have assumed that the filler material of the tailing edge is not contributing to the stiffness. 


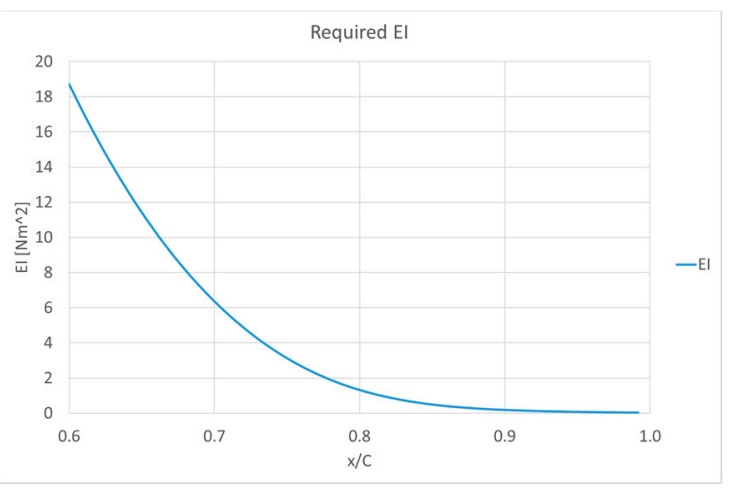

(a)

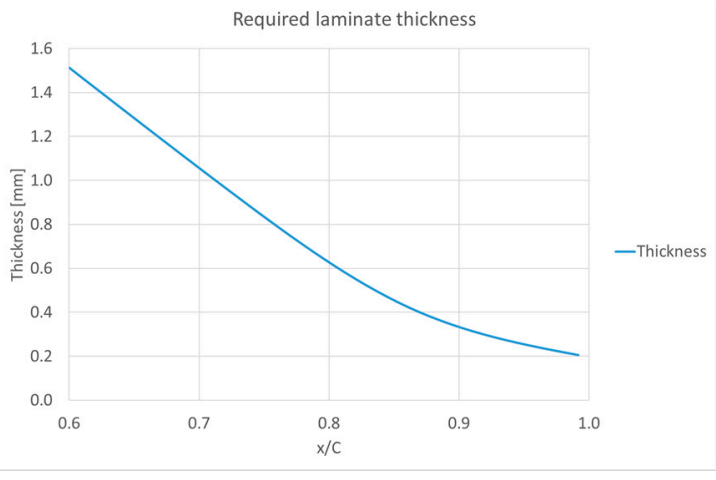

(b)

Figure 10. (a) Required rigidity (EI) and (b) required thickness of the foil trailing edge in order to map the required camber.

Now that the stiffness is known, and within hypothesis of linearity, we can apply (subtract) the load distribution at P1 to know the change of camber occurred between the unloaded shape and shape at P1, finally obtaining the design mould shape.

\subsection{Optimization of a flexible trailing edge}

Airfoil shape optimization is a critical activity documented in a large number of scientific papers and particularly the design of airfoils for sailing yachts have some specificity as reported in $[6,7]$ where the optimization of deformable composite wing was also described.

However, the special case of the hydrofoil present specific challenges that only a formalized optimization procedure can ensure a feasible result.

As it happens for any lifting surfaces the ability to lift a given load can be achieved by sizing the chord of the airfoil while the minimization of the drag coefficient may increase the efficiency of the system. In the case of the hydrofoil the risk of cavitation adds a heavy constrain to the optimization task.

To design the external shape of the airfoil and its internal structure a hierarchical nested optimization is needed even if it makes sense to decouple the problem as illustrated in the previous chapters where the rigidity of the trailing edge is imposed on the basis of load constraints on the airfoil.

The external optimization problem variables, once the two reference operating conditions corresponding to hydrofoil take-off and cruise speed condition are fixed, are the same of a classical airfoil shape definition, for example the coordinates of Bezier control points while the objectives are the drag at different operating points that has to be minimized. Chord length and incidence angle are determined by the load in one operating condition while the trailing edge structural rigidity, assumed to be linear, is defined by the internal optimization loop where the variables are the one defining the stiffness of the flexible trailing edge.

Fig 11 shows a schematic workflow built with modeFRONTIER [8] multidisciplinary design optimization software to solve the nested optimization task.

In this paper the optimization has been limited to a 2D case where the fluid dynamics characteristics of the airfoil are determined by the well-known simulation software XFoil [9] solver and the structural model is a simple analytical function of a flexible beam with constant cross section but the logic of the optimization loop could be used also for a complete 3D hydrofoil with sophisticated composite structure.

The objectives of the optimization are therefore the drag of the airfoil at the two design point conditions while shape, lift and drag in the complete operational envelope of the airfoil, even at rest, are a consequence of the selected deign points and can be consequently be calculated externally to the optimization loop. 


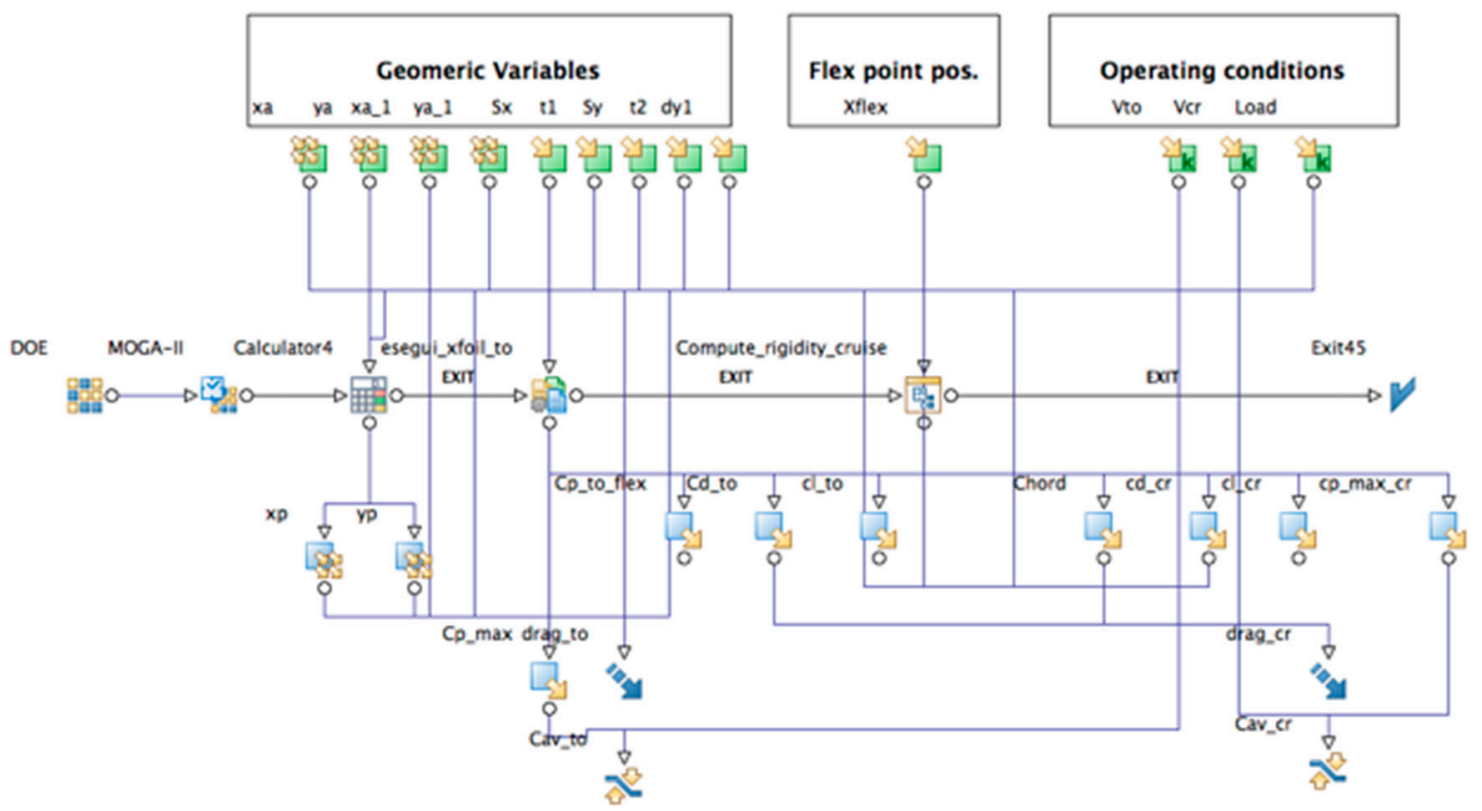

Figure 11. Optimization workflow built in modeFRONTIER software;

\section{Conclusion}

Hydrofoiling is having a vast success and fast development in nautical sports. Kitefoiling if probably the niche having the major expansion. The designers are chasing for foil concepts having the best possible performances, but being at the same time easier to sail. Still, foil design requires compromises, as the flow behavior is very different between the takeoff and the top speed. To adapt to these different flow regimes, ideally we would like to modify the foil section at any speed variation, that means having what is normally called a morphing wing. While a complete morphing of the foil is far from the possibility of existing technology, in this paper we have shown that at least one of the main parameter affecting foil characteristics, the camber, can the morphed and in a purely passive way just choosing the right stiffness properties of the trailing edge. This approach requires a designed procedure that couples hydrodynamic and structural characteristics of the foil (FSI) and can be optimized in the framework of a multidisciplinary design optimization software as modeFRONTIER.

\section{References}

1. Gordon Baker, Hydrofoil System for boats, US Patent US2856879A 1958

2. J. Grogono, ICARUS, the Boat that Flies. Adlar Coles Nautical; 1987, ISBN-10 0229118038.

3. F. Bethwaite, Higher Performance Sailing. Adlar Coles Nautical, 2008, pp. 52-54 (The Ilett Flying Moth), ISBN 978-1-4081-0126-1.

4. The Jacob's Ladder Project, http://www.panduj.plus.com/jladder/jl.htm accessed on 10 May 2017

5. I. H. Abbott, A. E. Von Doenhoff. Theory of Wing Sections, Dover Editions, 1949, ISBN-13:978-0-486-60586-9

6. Carlo Poloni, Andrea Giurgevich, Luka Onesti, Valentino Pediroda, Hybridization of a multi-objective genetic algorithm, a neural network and a classical optimizer for a complex design problem in fluid dynamics. CMAME 2000, Volume 186, Issue 2-4, pp.403-420, ISSN 0045-7825 
7. V. Pediroda, C. Poloni, Genetic Algoritms - Basics, Multi-criteria Optimization and Constraints Handling. In Optimistic - Optimization in Marine Design. Ed. Birk, Harries. pp. 83-111, Mensch \& Buch Verlag, ISBN: 38982051422003

8. modeFRONTIER v.2016 www.esteco.com

9. M. Drela , XFOIL: An Analysis and Design System for Low Reynolds Number Airfoils,,Lecture Notes in Engineering, vol.54, 1989 Springer Verlag 